\title{
A new maximum likelihood blood velocity estimator incorporating spatial and temporal correlation
}

Schlaikjer, Malene; Jensen, Jørgen Arendt

Published in:

Proceedings on IEEE Ultrasonics Symposium

Link to article, DOI:

10.1109/ULTSYM.2001.991990

Publication date:

2001

Document Version

Publisher's PDF, also known as Version of record

Link back to DTU Orbit

Citation (APA):

Schlaikjer, M., \& Jensen, J. A. (2001). A new maximum likelihood blood velocity estimator incorporating spatial and temporal correlation. In Proceedings on IEEE Ultrasonics Symposium (Vol. 2, pp. 1433-1437). IEEE. https://doi.org/10.1109/ULTSYM.2001.991990

\section{General rights}

Copyright and moral rights for the publications made accessible in the public portal are retained by the authors and/or other copyright owners and it is a condition of accessing publications that users recognise and abide by the legal requirements associated with these rights.

- Users may download and print one copy of any publication from the public portal for the purpose of private study or research.

- You may not further distribute the material or use it for any profit-making activity or commercial gain

- You may freely distribute the URL identifying the publication in the public portal 


\title{
A new maximum likelihood blood velocity estimator incorporating spatial and temporal correlation
}

\author{
Malene Schlaikjer and Jørgen Arendt Jensen \\ Center for Fast Ultrasound Imaging, Ørsted•DTU, Build. 348, \\ Technical University of Denmark, DK-2800 Kgs. Lyngby, Denmark
}

\begin{abstract}
The blood flow in the human cardiovascular system obeys the laws of fluid mechanics. Investigation of the flow properties reveals that a correlation exists between the velocity in time and space. The possible changes in velocity are limited, since the blood velocity has a continuous profile in time and space. This paper presents a new estimator (STC-MLE), which incorporates the correlation property. It is an expansion of the maximum likelihood estimator (MLE) developed by Ferrara et al. With the MLE a cross-correlation analysis between consecutive RF-lines on complex form is carried out for a range of possible velocities. In the new estimator an additional similarity investigation for each evaluated velocity and the available velocity estimates in a temporal (between frames) and spatial (within frames) neighborhood is performed. An a priori probability density term in the distribution of the observations gives a probability measure of the correlation between the velocities. Both the MLE and the STC-MLE have been evaluated on simulated and in-vivo RF-data obtained from the carotid artery. Using the MLE $4.1 \%$ of the estimates deviate significantly from the true velocities, when the performance is evaluated on the simulated data. These deviating estimates arise, as the search range in the correlation analysis exceeds one wavelength. By performing a similar investigation with the STC-MLE, no highly deviating estimates occur. The allowed search range is therefore larger with the STC-MLE. The performance evaluation on in-vivo data further reveals that the number of highly deviating velocity estimates in the tissue parts of the RF-signals are reduced with the STC-MLE. In general the resulting profiles are continuous and more consistent with the true velocity profile, and the introduction of the correlation property has improved the estimates.
\end{abstract}

\section{Introduction}

Estimation of the blood velocity distribution in the cardiovascular system is possible with medical ultrasound. Several blood velocity estimators have been developed in the past.
The shift in scatterer position is determined by means of the phase [1] or the time shift [2], [3], [4], [5] between a number of consecutive RF-signals. All the estimators split up the RF-signals in a number of segments. The estimation is then carried out on each of these segments individually. The aspect of correlation among the velocities in a spatial (within frame) and a temporal (between frames) neighborhood of the segment under investigation is not considered. The theory of fluid mechanics [6] predicts that the flow profile is continuous and smooth for non-turbulent flow. A highly negative velocity in a given position is unlikely, if its neighbors in time and space are highly positive. This correlation feature should be exploited and incorporated into the velocity estimation. This paper presents a maximum likelihood estimator, which incorporates the correlation property. Ferrara et al. [4], [5] have developed a maximum likelihood estimator, and the new statistical estimator represents an expansion of this. The expansion consists of an introduction of an a priori probability density, which describes the correlation to estimates adjacent in time and space.

\section{Maximum likelihood estimator}

The estimator by Ferrara et al. uses the principles of detection and estimation employed in radars. Given the knowledge of what signal to look for, one can determine the location and movement of an object through a correlation analysis between the known signal $s$ and a set of acquired signals $r_{l}$ [4], [5], [7], [8]. The parameter $l$ is the signal number in the acquired set. The presence of an object is determined through a maximum likelihood analysis. A brief discussion of the estimation theory used in radars is given in the following, and subsequently expanded to apply for ultrasound signals.

In radars the presence of an object is not known a priori, so the acquired signals either contain noise only, or $s$ with additive noise at some temporal location $\tau$ in the acquired signal. This represents a two hypothesis case in statistics and is referred to by $H_{0}$ and $H_{1}$, respectively. The dimensionality of the signal vectors, $r_{l}$, are reduced through a correlation com- 
putation to ease the subsequent analysis. The correlation is performed for a range of discrete values of the temporal shifts, as the final goal is to determine the location and movement of an object:

$$
q\left(n_{\tau}\right)=\sum_{l=1}^{N_{l}} \sum_{n=0}^{N_{s}-1} s(n) r_{l}^{*}\left(n_{\tau} l+n\right),
$$

where $n_{\tau}$ represents the shift in number of samples. The parameter $N_{l}$ is the total number of RF-signals acquired for the velocity estimation for one image line, $n$ represents the sample number within the signal, and $N_{s}$ is the total number of samples used in the correlation. In this study both $s(n)$ and $r(n)$ are complex signals. The detection and estimation theory gives that the position and velocity of the object can be determined by computing the likelihood ratio [7], [8]:

$$
\operatorname{LR}\left(q\left(n_{\tau}\right)\right)=\frac{p_{q \mid H_{1}}\left(q \mid H_{1}\right)}{p_{q \mid H_{0}}\left(q \mid H_{0}\right)}
$$

for a range of shifts $n_{\tau}$. The two terms $p_{q \mid H_{1}}\left(q \mid H_{1}\right)$ and $p_{q \mid H_{0}}\left(q \mid H_{0}\right)$ represent the probability of observing $q$ under the two hypothesis. The maximum value of (2) with respect to $n_{\tau}$ gives the estimate of $\hat{n}_{\tau}$ [7], [8], and thereby the position and velocity of the object:

$$
\hat{n}_{\tau}=\underset{n_{\tau}}{\arg \max }\left(L R\left(q\left(n_{\tau}\right)\right)\right)
$$

In medical ultrasound several objects in the form of tissue and blood scatterers are present. Still the above described approach can be employed. The estimation of the movement of a group of scatterers is the goal. The acquired signals have to be segmented, and the estimation must be carried out for each segment. One problem arises, as the composition of the underlying medium is unknown, and no explicit model for the acquired in-vivo RF-signal can be depicted a priori. The signal $s$ is therefore not given a priori. Instead a signal segment from one of the acquired RF-signals is used as the model for $s$, and the correlation computation becomes:

$$
q(v, M)=\sum_{l=1}^{N_{l}} \sum_{n=0}^{N_{s}-1} r_{1}\left(n+N_{s} M\right) r_{l}^{*}\left(n+N_{s} M+n_{\tau}\right),
$$

where $M$ is the segment number. The shift $n_{\tau}$ is related to the velocity $v$ through $v=n_{\tau} c f_{p r f} / 2 f_{s}$. The variables $c, f_{p r f}$, and $f_{s}$ represent the sound velocity, the pulse emission frequency, and the sampling frequency. Estimation of the velocity requires the computation of (2) for a range of velocities. U1trasound RF-signals are Gaussian distributed with zero mean and a variance [4]. The probabilities are:

$$
\begin{aligned}
& p_{q \mid H_{1}}\left(q \mid H_{1}\right)=\frac{1}{\pi\left(E+N_{0}\right) N_{l}} \exp \left(-\frac{|q(v)|^{2}}{\left(E+N_{0}\right) N_{l}}\right) \\
& p_{q \mid H_{0}}\left(q \mid H_{0}\right)=\frac{1}{\pi N_{0} N_{l}} \exp \left(-\frac{|q(v)|^{2}}{N_{0} N_{l}}\right) .
\end{aligned}
$$

The variable $E$ is the energy within the segment of the acquired signal without any noise present, and $N_{0}$ represents the energy of the noise in the segment. By inserting (5) into (2), taking the logarithm, and eliminating constant terms a simple expression for the sufficient statistic arises. Maximization of this gives the velocity estimate:

$$
\hat{v}(M)=\underset{v}{\arg \max }\left(|q(v, M)|^{2}\right) .
$$

The method described so far represents the maximum likelihood estimator (MLE) by Ferrara et al.

As stated in the introduction the velocities in a spatial and temporal neighborhood of the signal segment under investigation are correlated. The velocities are similar (not identical), as the velocity profile must be continuous and smooth. If the velocities in a spatial and temporal neighborhood are known, the velocity in the segment under investigation is restricted to lie within a range of velocities specified by the velocities of its neighbors. This information is exploited by expanding the probability density under $H_{1}$ by an a priori density $p_{a}$ :

$$
p(q, v(x, z, I))=p_{a}(v(x, z, I)) p_{q \mid H_{1}}\left(q \mid H_{1}\right) .
$$

A 2D image grid is introduced to distinguish the position $(x, z)$ under investigation from its neighbors. The lateral and axial positions are indicated by $x$ and $z$, respectively. The temporal neighborhood is represented by the image number I. Incorporation of the a priori knowledge requires a measure of similarity. The difference in velocity of two neighbors is used here, as a small difference resembles similarity:

$$
\begin{aligned}
& v_{d x}(x, z, I)=v(x+\Delta x, z, I)-v(x, z, I) \\
& v_{d z}(x, z, I)=v(x, z+\Delta z, I)-v(x, z, I) \\
& v_{d t}(x, z, I)=v(x, z, I-1)-v(x, z, I) .
\end{aligned}
$$

The variable $\Delta$ determines the spatial distance between neighbors. A histogram analysis of $v_{d x}, v_{d z}$, and $v_{d t}$ on simulated data (see Section 3), where the true velocity is known for a set of 2D CFM-mode images, gives the probability densities shown in Fig. 1. The densities of the differences are close to being Gaussian shaped, and in this study a Gaussian distribution is employed for $p_{a}$ :

$$
\begin{aligned}
p_{a}(v(x, z, I))= & \frac{1}{Z} \exp \left(-\Gamma_{1} \frac{E\left(E+N_{0}\right)}{N_{0}} C_{1}(x, z, I)\right. \\
& \left.-\Gamma_{2} \frac{E\left(E+N_{0}\right)}{N_{0}} C_{2}(x, z, I)\right) .
\end{aligned}
$$

The parameter $Z$ is a normalization constant, which assures that $\int p(q, v) d q d v_{d x} d v_{d z} d v_{d t}=1$. The parameters $\Gamma_{1}$ and $\Gamma_{2}$ are scaling factors, and are related to the variances of the temporal and spatial distributions. The terms $C_{1}$ and $C_{2}$ contains 


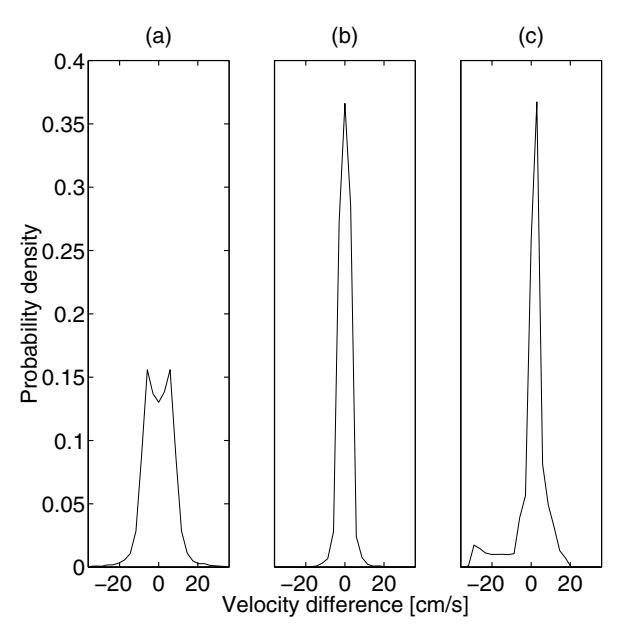

Figure 1: Probability densities of the axial, $v_{d z}$ (a), lateral, $v_{d x}$ (b), and temporal, $v_{d t}$ (c) variations in the $2 \mathrm{D}$ velocity profiles.

the similarity investigation:

$$
\begin{aligned}
C_{1}(x, z, I)= & (\hat{v}(x, z, I-1)-v(x, z, I))^{2} \\
& +(\hat{v}(x+\Delta x, z, I-1)-v(x, z, I))^{2} \\
& +(\hat{v}(x, z+\Delta z, I-1)-v(x, z, I))^{2} \\
C_{2}(x, z, I)= & (\hat{v}(x-\Delta x, z, I)-v(x, z, I))^{2} \\
& +(\hat{v}(x, z-\Delta z, I)-v(x, z, I))^{2} .
\end{aligned}
$$

The previously estimated velocities in the neighborhood are named $\hat{v}$ to distinguish them from the velocity $v$ currently being evaluated for the segment in $(x, z)$. The choice of neighbors rely on which estimates are available at the time of the estimation of the velocity in $(x, z)$. Again, by taking the logarithm, rearranging terms, and removing constant terms a sufficient statistic results from which the velocity estimate can be obtained:

$$
\begin{aligned}
\hat{v}(x, z, I)= & \underset{v}{\arg \max }\left(\frac{1}{N_{l}\left(E+N_{0}\right)^{2}}|q(v, x, z(M))|^{2}\right. \\
& \left.-\Gamma_{1} C_{1}(x, z, I)-\Gamma_{2} C_{2}(x, z, I)\right) .
\end{aligned}
$$

The velocity estimate is determined by performing a correlation analysis along with a similarity investigation for a range of velocity values. The expanded maximum likelihood estimator is named the STC-MLE, where STC refers to the incorporation of the spatial and temporal correlation.

\section{Results}

The STC-MLE has been tested on simulated and in-vivo data. The simulated data were generated with Field II [9]. An apodization and focusing scheme matching a $5 \mathrm{MHz}$ linear

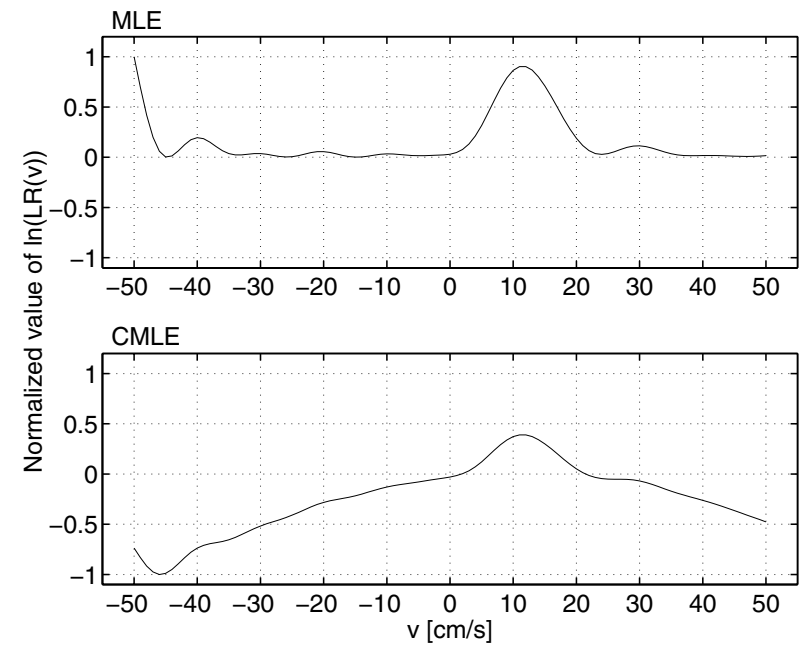

Figure 2: The value of the sufficient statistic for a range of velocities for the MLE (top) and the STC-MLE (bottom).

array connected to a B-K Medical A/S 3535 scanner was implemented. The data cover three cardiac cycles, and the excitation pulse extends 1.5 cycles. Womersleys pulsatile flow profile and tissue motion due to pulsation were implemented [10], [11], [12]. The SNR is $20 \mathrm{~dB}$. The in-vivo data were sampled with a dedicated sampling system [13] interfaced to a B-K Medical A/S 2102 scanner using a $5 \mathrm{MHz}$ linear array transducer. Ten seconds of data were acquired. A 5 cycles excitation pulse is emitted. The RF-data were acquired at the carotid artery for both data sets. The vessel lies an angle of $35^{\circ}$. A total of 8 and $9 \mathrm{RF}$-signals are used by the estimator for the simulated and in-vivo data, respectively.

\subsection{Results with simulated data}

Estimators, which solely employ the cross-correlation function to determine the velocity, can encounter problems due to the segmentation of the RF-signals. Multiple peaks in the cross-correlation can occur, and the maximum of the correlation function will not necessarily occur at the true velocity. Usually this problem is overcome by limiting the search range to a temporal interval equal to one ultrasound wavelength. Unfortunately the range of velocities, which can be estimated, is then also limited. By incorporating the correlation between the velocities in a neighborhood one can hope that this problem can be minimized. Inspection of the estimated velocity profiles obtained with the MLE, when the search range has not been limited, confirms that highly deviating estimates occur. These are a result of the problem of the cross-correlation analysis. An example hereof is plotted in Fig. 2. The maximum of the likelihood ratio is obtained at a velocity of $-0.5 \mathrm{~m} / \mathrm{s}$. A second peak is located at $0.11 \mathrm{~m} / \mathrm{s}$, 
which is close to the true velocity of $0.13 \mathrm{~m} / \mathrm{s}$. The MLE thus produces an incorrect velocity estimate. The problem can be resolved with the STC-MLE. The best choice of the scale parameters $\Gamma_{1}$ and $\Gamma_{2}$ has been investigated for this purpose. By increasing the values of the scale parameters $\Gamma_{1}$ and $\Gamma_{2}$ the information available from the neighboring estimates gets to play a bigger role in the estimation. All highly deviating estimates are removed, if $\left(\Gamma_{1}, \Gamma_{2}\right) \geq(2,2)$. The likelihood ratio for the STC-MLE in Fig. 2 shows that only one peak is present, as a velocity of $-0.5 \mathrm{~m} / \mathrm{s}$ is far from the velocities in the temporal and spatial neighborhood, and it is therefore unlikely. The STC-MLE estimator makes it possible to expand the search range without encountering the problems introduced by the cross-correlation analysis. In Fig. 3 a set of $2 \mathrm{D}$ velocity profiles obtained with the MLE and STC-MLE are plotted. A total of $4.1 \%$ of the velocity estimates computed with the MLE deviate significantly, whereas none is obtained with the STC-MLE. As the true velocities are known, the root-mean-square (RMS) error is computed as a measure of performance. The RMS error for MLE and STC-MLE is 0.21 and $0.12 \mathrm{~m} / \mathrm{s}$, respectively, so an improvement of $43 \%$ is obtained with the STC-MLE. The majority of the improvement is due to the removal of the highly deviating estimates.

\subsection{Results with in-vivo data}

The velocity profiles have been estimated for a range of values of $\Gamma_{1}$ and $\Gamma_{2}$, just as was the case with the simulated data. The values of $\Gamma_{1}$ and $\Gamma_{2}$ has been further increased to improve on the estimation with respect to obtaining profiles, which are more continuous and smooth as expected. The smoothening effect is obtained as shown in Fig. 4. The scale parameters are $\left(\Gamma_{1}, \Gamma_{2}\right)=(100,100)$. The carotid artery is located at a depth of $2.4 \mathrm{~cm}$ for the lateral position $0 \mathrm{~cm}$. Highly deviating estimates are also produced by most estimators in the part of the signal, which represents an interaction with tissue. This is due to the presence of noise in the RF-signals, and usually some post-processing is performed to remove them prior to display. With the STC-MLE estimator less highly deviating estimates are obtained in the tissue region. Again, the introduction of the correlation feature of the velocities in a spatial and temporal neighborhood, improves on the estimation.

\section{Conclusion}

The properties of fluid flow can successfully be incorporated into a blood velocity estimator based on maximum likelihood, and improves the basis for the estimation. The resulting velocity profiles are more smooth. Additionally the STC-MLE has the advantage that the number of highly deviating velocity estimates in the tissue regions are lowered. Secondly, the velocity range that can be investigated with the STC-MLE
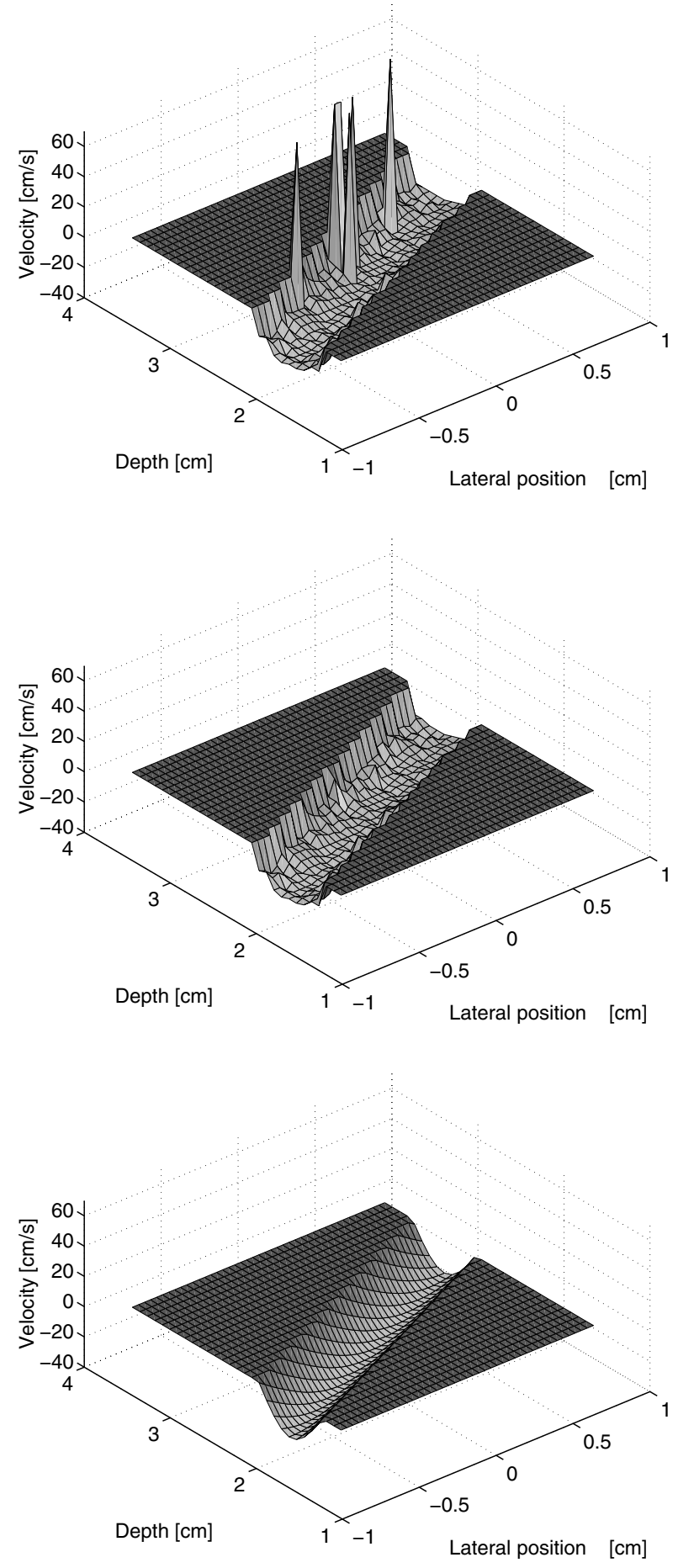

Figure 3: The estimated velocity profile with MLE (top) and STC-MLE (middle) for a SNR of $20 \mathrm{~dB}$ on simulated data. The scale parameters for the STC-MLE are $\left(\Gamma_{1}, \Gamma_{2}\right)=(2,2)$. The true velocity profile is shown in the bottom plot. 

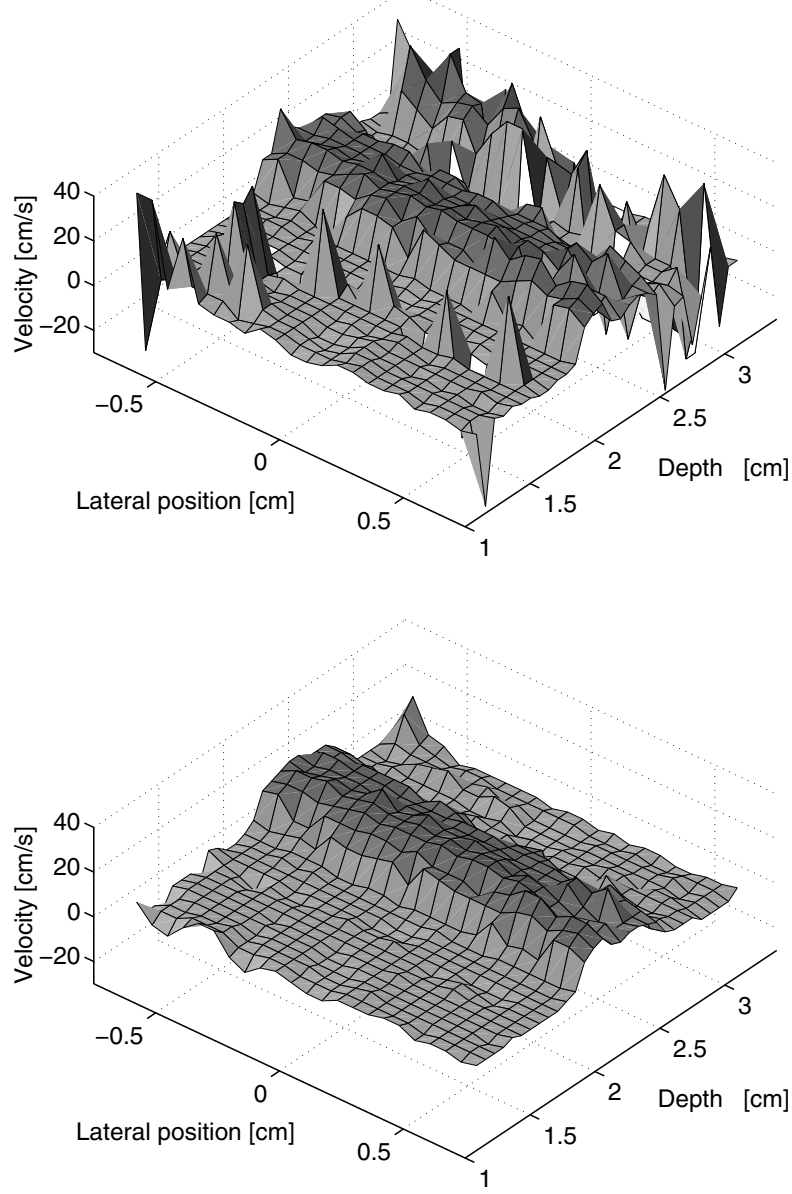

Figure 4: The velocity profile obtained with the MLE (top) and the STC-MLE (bottom) on in-vivo data. The scale parameters are $\left(\Gamma_{1}, \Gamma_{2}\right)=(100,100)$.

spans a larger range compared to other estimators based on a correlation analysis.

\section{Acknowledgement}

This work was supported by grant 9700883,9700563 , and 5013-00-0025 from the Danish Science Foundation, grant 980018-311 from the Technical University of Denmark, and by B-K Medical A/S, Gentofte, Denmark.

\section{References}

[1] C. Kasai, K. Namekawa, A. Koyano, and R. Omoto. Real-time two-dimensional blood flow imaging using an autocorrelation technique. IEEE Trans. Son. Ultrason., 32:458-463, 1985.
[2] S. K. Alam and K. J. Parker. The butterfly search technique for estimation of blood velocity. Ultrasound Med. Biol., 21:657-670, 1995.

[3] J. A. Jensen. Estimation of Blood Velocities Using Ultrasound: A Signal Processing Approach. Cambridge University Press, New York, 1996.

[4] K. W. Ferrara and V. R. Algazi. A new wideband spread target maximum likelihood estimator for blood velocity estimation - Part I: Theory. IEEE Trans. Ultrason., Ferroelec., Freq. Contr., 38:1-16, 1991a.

[5] K. W. Ferrara and V. R. Algazi. A new wideband spread target maximum likelihood estimator for blood velocity estimation - Part II: Evaluation of estimators with experimental data. IEEE Trans. Ultrason., Ferroelec., Freq. Contr., 38:17-26, 1991b.

[6] R. W. Fox and A. T. McDonald. Introduction to fluid mechanics. John Wiley and Sons, 1985.

[7] H. L. Van Trees. Detection, Estimation and Modulation Theory, Part I: Detection, estimation, and linear modulation theory. Wiley, 1971.

[8] H. L. Van Trees. Detection, Estimation and Modulation Theory, Part III: Radar-sonar signal processing and Gaussian signals in noise. Wiley, 1971.

[9] J. A. Jensen. Field: A program for simulating ultrasound systems. Med. Biol. Eng. Comp., 10th NordicBaltic Conference on Biomedical Imaging, Vol. 4, Supplement 1, Part 1:351-353, 1996b.

[10] M. Schlaikjer. Development and characterization of algorithms for estimation of blood velocity with ultrasound. PhD thesis, Ørsted•DTU, Technical University of Denmark, Kgs. Lyngby, Denmark, 2001.

[11] W. W. Nichols and M. F. O'Rourke. McDonald's Blood Flow in Arteries, Theoretical, Experimental and Clinical Principles. Lea \& Febiger, Philadelphia, 1990.

[12] M. Schlaikjer, S. Torp-Pedersen, J. A. Jensen, and P. F. Stetson. Tissue motion in blood velocity estimation and its simulation. In Proc. IEEE Ultrason. Symp., pages 1495-1499, 1998.

[13] J. A. Jensen, O. Holm, L. J. Jensen, H. Bendsen, H. M. Pedersen, K. Salomonsen, J. Hansen, and S. Nikolov. Experimental ultrasound system for real-time synthetic imaging. In Proc. IEEE Ultrason. Symp., volume 2, pages 1595-1599, 1999. 